\title{
The Eighth International Symposium on Plecoptera, Toulouse, August 17-20, 1983
}

Forty one registrants from nineteen countries attended the Symposium. The opening address was delivered by Prof. H.B.N. Hynes and the closing remarks by Prof. J. Aubert. Dr. P. Zwick said a few words in memory of the late Prof. J. Illies'. Mr. I.D. McLellan gave an informal talk on his researches on New Zealand stoneflies and twenty seven papers were presented during six sessions. These papers are published in the present volume except the following one : I. Sivec, "Present state of Chinese stoneflies researches $"$.

On Friday August 19 th, the participants and the accompanying persons visited the Cave Laboratory of the C.N.R.S. at Moulis and collected some stoneflies in the Mas d'Azil Cave, near the River Arize, and at about $1000 \mathrm{~m}$ a.s.l. in the Bethmale Valley (Cen. tral Pyrenees).

\section{Acknowledgments}

I would like to express my gratitude to the following persons and institutions :

Dr. R.W. Baumann and Dr. P. Zwick sent me the mailing list of the Perla Newsletter. The sessions were held in Prof. C. Leredde's laboratory which. incidentally, was previously that of a Plecopterist, the late Prof. $\mathbf{R}$. Despax. Prof. E. Angelier and Mrs. M. Soulié took part in the organization. The help of Dr. A. Thomas and M.Z. Moubayed during the whole meeting was invaluable. Dr.C. Juberthie, Director of the Laboratoire Souterrain du C.N.R.S. at Moulis, organized a visit of the Cave Laboratory and a projection of a film on karst hydrology. Prof. J. Auberi, Prof. H.B.N. Hynes, Prof. T. Kawai, Prof. K.W. Stewart and Dr. P. Zwick acted as section leaders. Dr. P.P. Harper revised the English of some manuscripts. The National Center for Scientific Research (C.N.R.S.), the University Paul Sabatier, the Municipal Council of Toulouse and the General Council of the Haute-Garonne Department provided their financial support.

C. Berthélemy

1. For an obituary notice, see Aquatic Insects, vol. 4 (1982). $n^{\circ} 3: 121 \cdot 124$.

\section{List of registrants}

Dr. J. Alba Tercedor. Departamento de Zoologia. Facultad de Ciencias, Universidad de Granada, España.

Dr. N. Alouf, Département des Sciences Naturelles. Faculté des Sciences I, Université Libanaise, Hadath Beyrouth, Liban.

Prof. E. Angelier, Laboratoire d'Hydrobiologie, Université Paul Sabatier, 118, route de Narbonne, F.31062 Toulouse Cedex, France.

Prof. J. Aubert, Ch. des Toises 1, CH - 1095 Lutry, Suisse.

Prof. C. Berthélemy, Laboratoire d'Hydrobiologie, Université Paul Sabatier, 118, route de Narbonne, F-31062 Toulouse Cedex, France.

M. Boumaiza, Laboratoire de Biologie Animale et Ecologie, Faculté des Sciences, Campus Universitaire du Belvédère, Tunis, Tunisie.

Dr. J.E. Brittain, Zoologisk Museum, Universitetet i Oslo, Sars Gt. 1, Oslo 5, Norway.

M. Britton, Mainzer Kolleg Zi. Nr. 160, Jakob Welder-Weg B, D - 6500 Mainz, B.R.D.

M.J. Costello, c/o Zoology Department, Lee Maltings, Prospect Row, University College, Cork, Eire.

B. Dumont, C.E.M.A.G.R.E.F., Le Tholonet, B.P. 99, F-13603, Aix-en-Provence, France.

Dr. C.G. Froehlich, Departamento de Biologia, FFCLRP, Universidade de São Paulo, 14100 Ribeirāo Preto, SP, Brasil.

Dr. D. Garcia de Jalón, Departamento de Zoologia y Entomologia, E.T.S. de Ingenie ros de Montes, Ciudad Universitaria, Madrid 3, España.

Dr. Gonzàlez del Tánago, Laboratorio de Hidrologia, E.T.S. de Ingenieros de Montes, Ciudad Universitaria, Madrid 3, Espana.

Dr. F. Harper and Dr. P.P. Harper, Département de Sciences Biologiques, Université de Montréal, C.P. 6128 , Succursale " A n. Montreal, Québec, H3C 3J7, Canada.

Prof. H.B.N. Hynes, Department of Biology, University of Waterloo, Ontario N2L 3Gl, Canada.

Dr. N.N. Kapoor, Biology Department, Concordia University, S.G.W. Campus, Montréal, Québec, H3G IM8, Canada.

Prof. Dr. T. Kawai, Biological Institute, Nara Women's University, Kitauoya nishi-machi, Nara City, 630 Japan.

Dr. W. Kittel, ul. Piekna $69 / 71$ m. 56, 93558 Lódź, Poland.

Dr. M. Kohno, T 965, 1.15 Óhatamachi, Aizu-Wakamatsu City, Fukushima Prefecture, Japan

Dr. K. Kuusela, Department of Zoology, University of Oulu, PL 191, 90101 Oulu 10, Finland. 

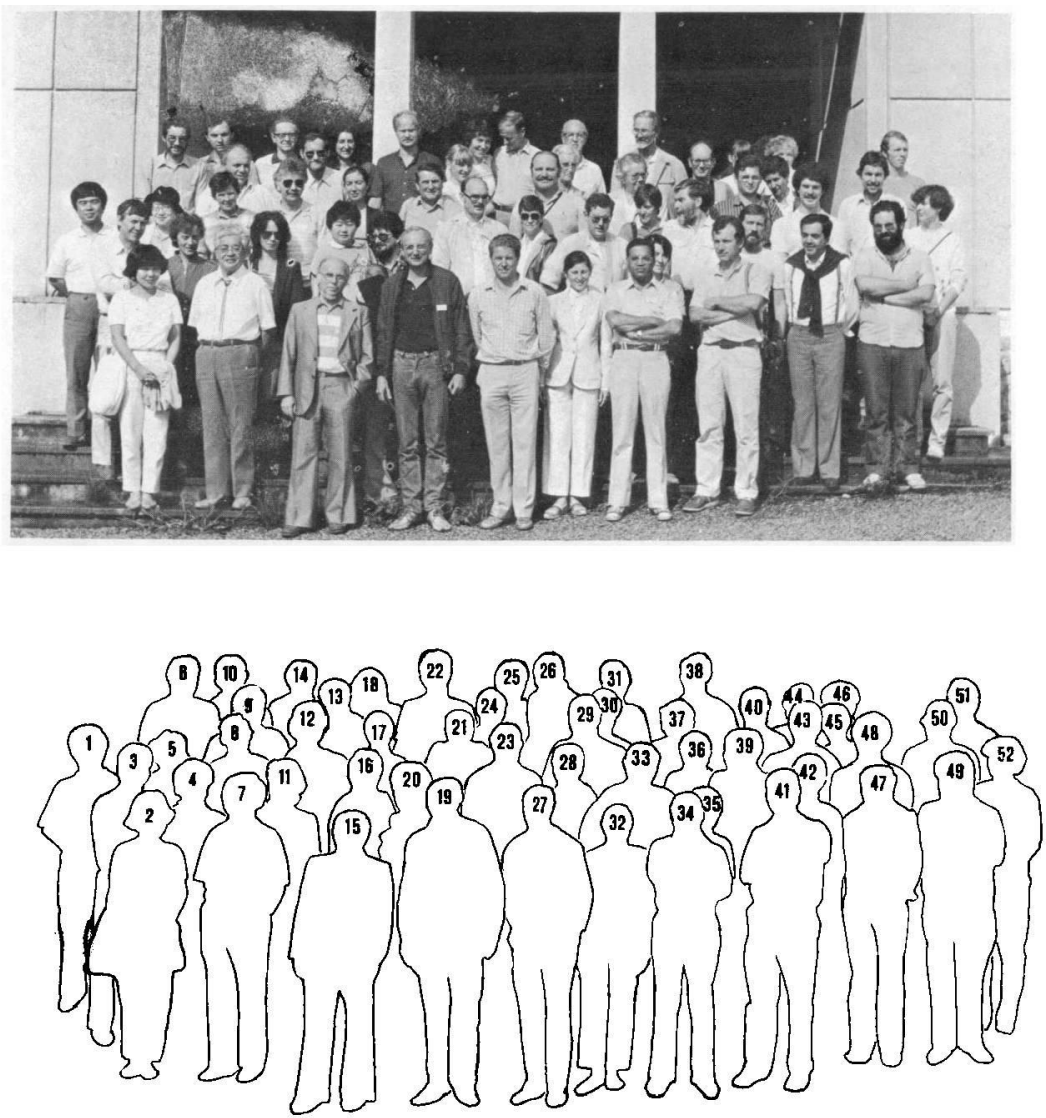

Fig. 1. Group picture in front of the Cave Laboratory at Moulis, 1, K. Tanida : 2, K. Kawai ; 3, A. Lillehammer : 4. M. Lilleham mer; 5, M. Kohno ; 6, J.E. Brittain ; 7, T. Kawai ; 8, H. Zwick ; 9. P. Zwick; 10, J. Alba ; 11, J. Ward ; 12, J.V. Ward ; 13, I. Sivec : 14. C.G. Froehlich; 15, C. Juberthie; 16, Y. Tanida; 17, M. Gonzàlez del Tánago; 18, M. Froehlich; 19. C. Berthélemy ; 20 D. de Jalón ; 21, 1.D. McLellan; 22, S.J. Saltveit ; 23, B.P. Stark; 24, C. Rupprecht ; 25, I. Rupprecht ; 26, R. Rupprecht, 27. A. Thomas ; 28 , L. Stark ; 29 , K.W. Stewart ; 30, M. Hynes; 31, H.B.N. Hyres; 32, F.B. Michaelis; 33, A. Sanchez Ortega 34 N.N. Kapoor: 35, M.A. Puig; 36, F. Harper; 37, C. Aubert ; 38, J. Aubert ; 39, P.P. Harper ; 40, C. Ravizza ; 41. B. Durnont : 42. W. Kittel ; 43, K. Kuusela ; 44, M. Rupprecht ; 45, E. Ravizza Denatteis ; 46, E. Seitz; 47. N. Alouf ; 48, M. Costello; 49 , P. Membiela ; 50, M. Marten; 51, M. Britton ; 52 . \$. Marten. 


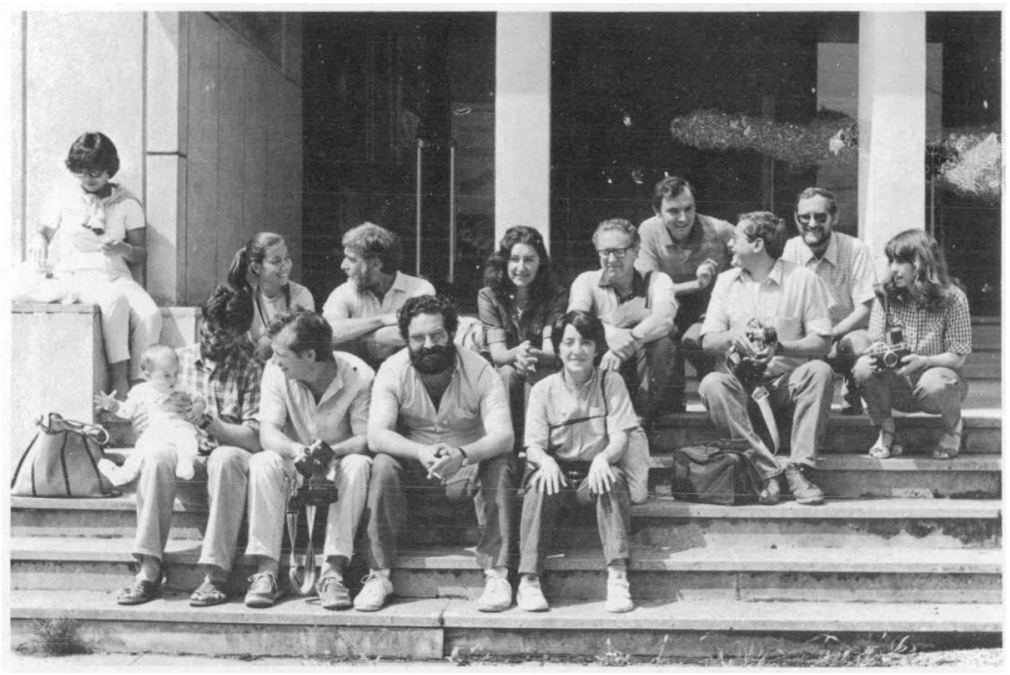

Fig. 2. From left to right, first row : D. de Jalón, B. Dumont, P. Membiela, M.A. Puig : second row : M. Gonzalez del Tánago, P.P. Harper, M. Froehlich, C. Froehlich, A. Sanchez-Ortega, I.R. Wais ; third row : K, Kawai, J. Alba, I. Sivec.

Dr. A. Lillehammer, Zoologisk Museum, Universitetet i Oslo, Sars Gt. 1, Oslo 5, Norway.

I.D. McLellan, P.O. Bux 95, Westport, New Zealand.

M. Marten, Limnologische Flussstation des Max.PlanckInstituts für Limnologie, Postfach 260, D - 6407 Schlitz, B.R.D.

P. Membelia Iglesia, I.N.B. a Otero Pedraio s c/Padre Feijoo 14. SP - 32005 Orense, Espana.

Dr. F.B. Michaelis, Nature Conservation Evaluation Branch, Australian National Park \& Wildlife Service, G.P.O. Box 636, Canberra. A.C.T. 2601, Australia

Z. Moubayed, Laboratoire d'Hydrobiologie, Université Paul Sabatier, 118, route de Narbonne, F-31062 Toulouse Cedex, France

Dr. M.A. Puig Garcia, Departament d'Ecologia, Fac. Biologia, Universitat de Barcelona, Av. Diagonal 645, Barcelona 28. España.

Dr. E. Ravizza, Largo Murani 4, I - 20133 Milano, Italia.

Dr. E. Ravizza Dermatteis, Dipartimento di Biologia, Via Celoria 26, I - 20133 Milano, Italia.

Prof. Dr. R. Rupprecht, Institut für Zoologie, Saarstrasse 21. Postfach 3980, D - 6500 Mainz, B.R.D.

Dr. S.J. Saltveit, Zoologisk Museum, Universitetet i Oslo, Sars Gt. 1, Oslo, 5, Norway.
A. Sanchez Ortega, Departamento de Zoologia, Facultad de Ciencias, Universidad de Granada, España.

Dr. I. Sivec, Prirodoslovni muzej Slovenije, Presernova 20. pp. 290, 61001 Ljubljana, Jugoslavija.

Dr. B.P. Stark, Department of Biology, Mississippi College, Clinton, MS 39058, U.S.A.

Prof. K.W. Stewart. Department of Biological Sciences, North Texas State University, Denton, Texas 76203 , U.S.A.

Dr. K. Tanida, Biological Laboratory, Faculty of General Science, Univ, Osaka Pref., Mozu-ume-machi, Sakai-shi, Osaka Pref., 591 Japan.

Dr. A. Thomas, Laboratoire d'Hydrobiologie, Université Paul Sabatier, 118, route de Narbonne, F-31062 Toulouse Cedex, France.

I.R. Wais de Bagden, Museo Argentino de Ciencias Naturales, Av, Angel Gallardo 470, CC 220 Suc. 5, 1405 Buenos Aires, Argentina.

Dr. J. Ward, Department of Zoology and Entomology, Colorado State University, Fort Collins, Colorado 80523 , U.S.A.

Dr, habil. P, Zwick, Limnologische Flussstation des MaxPlanck-Instituts für Limnologie, Postfach 260, D- 6407 Schlitz, B.R.D. 